\title{
A SIMPLE APPROACH FOR DESIGNING A FILTER ON MICROSTRIP LINES
}

UDC: 621.372 .543

Original scientific paper

https://doi.org/10.18485/aeletters.2019.4.1.3

\author{
Ashish Kumar ${ }^{1}$ \\ ${ }^{1}$ Aryabhatta research institute of observational sciences (ARIES), Nainital, India
}

\begin{abstract}
:
This paper deals with the design and fabrication of edge-coupled band pass filter (BPF) circuit (fifth order) on microwave laminate for $4.5 \mathrm{GHz} \pm 0.5 \mathrm{GHz}$ application. The design of filter is realized on a high quality RT/ duroid laminate having the dielectric constant 10.5 and substrate thickness 1.27 $\mathrm{mm}$. The relevant design specifications, simulation, and test results of the circuit is described. The numerical calculations and simulations are performed in Linpar. The Printed Circuit Board (PCB) artwork is prepared in CorelDraw. A prototype of the design was manufactured and tested on microwave network analyzer. Some offsets are observed between the theoretical and practical results, which may be attributed to the wide tolerance in the dielectric permittivity specified for the RT/ duroid substrate.
\end{abstract}

\author{
ARTICLE HISTORY \\ Received: 22.01.2019. \\ Accepted 26.03.2019. \\ Available: 31.03.2019.
}

\section{KEYWORDS}

filter, microstrips, edgecoupled, linpar, CorelDraw, microwave

\section{INTRODUCTION}

In electronic circuits, the filter is a network having non-uniform frequency response characteristics in the desired frequency range. They are used to manipulate the signal by enhancing or attenuating certain frequency ranges or bands, and sometimes, for rejecting harmonics and excluding out-of-band signal. The ideal filter network provides perfect transmission for all the frequencies in certain passband region and infinite attenuation in stopband regions. Such ideal characteristics can't be achieved in practice. Therefore, filter design aims at approximating the ideal characteristics within acceptable tolerances.

At microwave frequency, instead of the lumped element inductors and capacitors, the transmission line sections are used which behaves as inductors and capacitors. The microwave filters have a wide range of applications demanding high performance at reduced size and production cost [1]. These filters are primarily used in wireless handsets, satellite receivers and military systems [2]. The filters with high performance characteristics is achieved when designed using sophisticated planar or waveguide architecture, but at the cost of certain complexities. However, without entering into any design complexities, the present work is focussed on the basic steps involved in the design and fabrication of an edge-coupled BPF on microstrip lines. Following the steps, one can realize any other microwave filters for their frequency band of interests.

\section{THEORY}

\subsection{Coupled lines}

The coupled lines are formed when the two or more unshielded transmission lines are placed in close proximity to each other [3]. Likewise transformers, the power at one transmission line gets coupled to other. The level of coupling depends on mode and direction of electromagnetic wave propagation, physical and electrical dimensions of the line, and frequency of operation. The coupled lines may be in symmetrical or asymmetrical form. The symmetrical coupled line structures support odd and even mode excitations, and the interaction between these modes induces the coupling between the line conductors. The even and odd modes have different characteristics 
impedances (Zo_odd and Zo_even) from line to ground and represents the in-phase and out of phase characteristics of the driven source, respectively [4].

The theory of coupled line is utilized in the realization of numerous passive microwave components like filters, directional couplers, resonators, impedance transformers, and others, of which the design of filters on the planar coupled transmission lines such as microstrip lines are the most popular $[2,3,5]$ due to its ease in fabrication using low-cost RT/ duroid laminates and processes involved therein.

The planar transmission lines at microwave frequencies are broadly classified in two categories: (i) the one that supports a Transverse Electromagnetic Mode (TEM) of propagation, like coaxial line, stripline etc., or the one that supports quasi-TEM modes of propagation, and (ii) the one like hollow waveguides, dielectric guides etc. that does not support such modes of propagation. In this work, the planar coupled microstrip lines are used which supports the quasi- TEM of propagation [6]. The microstrip line is best known and widely used for microwave filter designs because of its inherent advantages like ease in integration with the active solid-state devices, good heat sinking, low cost, compact design and good repeatability. Fig. 1 shows the cross-sectional view of a microstrip line $[3,6]$. It contains a single dielectric substrate of gallium arsenide (GaAs), alumina $\left(\mathrm{Al}_{2} \mathrm{O}_{3}\right)$ or teflon material having the dielectric constant as $\varepsilon_{r}$.

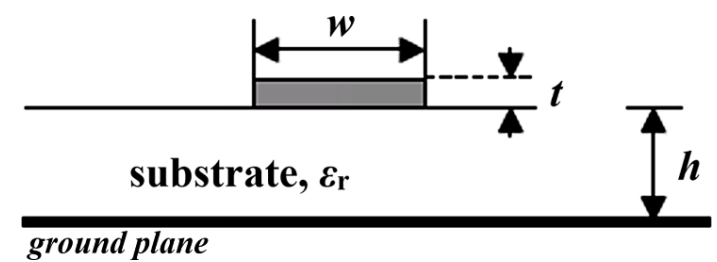

Fig. 1. Cross-sectional view of a microstrip line

The height, $h$ of the substrate is chosen to be very smaller than its wavelength. At the top of the dielectric substrate, there is a conducting strip of width, $w$ and thickness, $t$ and the bottom surface is the ground plane. The transmission characteristic of the microstrip line is well described by its effective dielectric constant, $\varepsilon_{\text {eff }}$ and its characteristic impedance, $Z_{o}$ expressed mathematically as $[3,7]$ :

$$
\varepsilon_{\text {eff }}=\frac{\varepsilon_{r}+1}{2}+\frac{\varepsilon_{r}-1}{2}\left(1+\frac{10}{(W / h)}\right)^{-0.564 a\left(\frac{\varepsilon_{r}-0.9}{\varepsilon_{r}+3}\right)^{0.053}}
$$

$$
Z_{o}=\frac{\eta}{2 \pi \sqrt{\varepsilon_{\text {eff }}}} \ln \left[\frac{F}{W / h}+\sqrt{1+\left(\frac{2}{W / h}\right)^{2}}\right]
$$

The terms, $a$ and $F$ are computed using the width-height ratio, $W / h$ of the microstrip laminate. The term, $\eta$ is the wave impedance in free space.

In microstrip line configuration, the transmission line conductor of a typical $N+1$ section of coupled line filter takes the form shown in Fig. 2a-b.
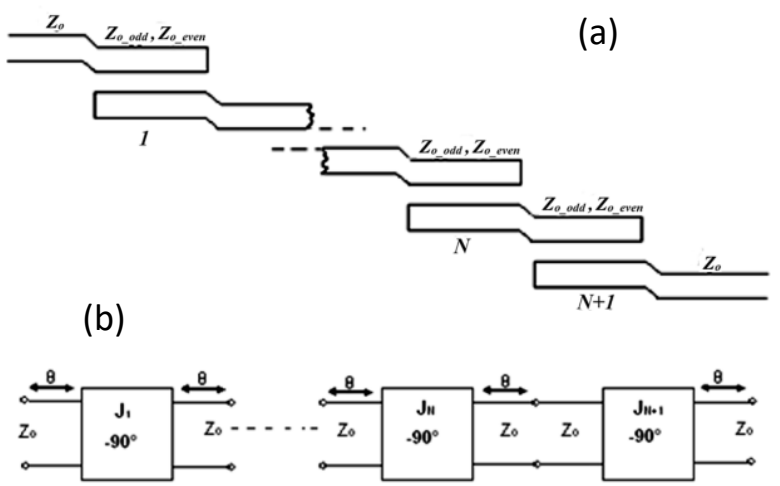

Fig. 2. Coupled line filter (a) layout of a typical $N+1$ section of coupled line band pass filter, and (b) its equivalent circuit.

\subsection{Filter parameters}

When dealing with filters, the following parameters plays an important role [2-3, 8]:

- Insertion Loss: The insertion loss quantifies the amount below the $0 \mathrm{~dB}$ line the power amplitude response drops. Ideally, a perfect filter inserted into RF circuit path would introduce no power loss in the passband, or it would have zero insertion loss. In reality however, certain amount of power loss associated with the filter is expected. Mathematically, Insertion Loss (I.L.) = $10 * \log \left(P_{\text {in }} / P_{\mathrm{L}}\right)=-10 * \log \left(1-\left|R C_{\text {in }}\right|^{2}\right)$, where, $P_{L}=$ power delivered to the load, $P_{\text {in }}=$ power from source, and $\left|R C_{\text {in }}\right|=$ reflection coefficient looking into the filter.

- Ripple: It is the difference between maximum and minimum amplitude response (in $\mathrm{dB}$ or Nepers) or the flatness of signal in the passband.

- 3dB-Bandwidth: For any filter, it is the difference between upper and lower frequencies typically recorded at the $3 \mathrm{~dB}$ attenuation points above the pass-band.

- Shape Factor: Shape factor defines the sharpness of the filter response, by taking the ratio between the $60 \mathrm{~dB}$ and the $3 \mathrm{~dB}$ bandwidth.

and, 
- Rejection: Attenuation level for the undesirable signal frequencies, however in reality an upper bound on this value called as rejection ratio, is expected. Practically $60 \mathrm{~dB}$ is the rejection rate.

\section{THEORY}

\subsection{Design specifications}

The edge-coupled BPF is designed with the specification as given in Table 1.

Table 1. Design specification of filter

\begin{tabular}{|c|c|}
\hline Parameters & Values \\
\hline bandwidth & $1 \mathrm{GHz}$ \\
\hline frequency range & $4-5 \mathrm{GHz}$ \\
\hline centre frequency $\left(f_{\mathrm{o}}\right)$ & $4.5 \mathrm{GHz}$ \\
\hline order of filter & Fifth Order \\
\hline approximation & Chebyshev \\
\hline ripple Factor & $0.1 \mathrm{~dB}$ \\
\hline dielectric constant & 10.5 \\
\hline substrate thickness & $1.27 \mathrm{~mm}$ \\
\hline
\end{tabular}

\subsection{Design steps}

The series of steps that were followed in designing the filter, as detailed below [3, 8-10]:

(a) Selection of standard filter coefficient: Based on the type and order of the filter (generally Butterworth or Chebyshev) needed, an appropriate filter coefficients $g_{0}, g_{1}, \ldots, g_{N}, g_{N+1}$ from the standard tables are selected. In this work, the filter coefficients corresponding to fifth order Chebyshev filter with $0.1 \mathrm{~dB}$ ripple is taken. Chebyshev filter is selected due to its narrow pass band response.

(b) Identification of normalized bandwidth, upper and lower cut-off frequencies: For the desired filter with lower and upper cut-off frequencies $\omega_{\mathrm{L}}, \omega_{\mathrm{U}}$, and the centre frequency $\omega_{0}\left[=\left(\omega_{L}+\omega_{U}\right) / 2\right]$, the normalized bandwidth can be defined as:

$$
\Delta=\left(\omega_{u}-\omega_{L}\right) / \omega_{0}
$$

(c) Coupling coefficients calculation: The coupling between the individual filter elements can be calculated using:

$$
\begin{gathered}
J_{01}=\frac{\sqrt{(\pi \Delta)}}{\left(Z_{o} \sqrt{\left(2 g_{0} g_{1}\right)}\right)}, J_{12}=\frac{(\pi \Delta)}{\left(2 Z_{o} \sqrt{\left(g_{1} g_{2}\right)}\right)} \\
J_{23}=\frac{(\pi \Delta)}{\left(2 Z_{o} \sqrt{\left(g_{2} g_{3}\right)},\right.}, J_{34}=\frac{(\pi \Delta)}{\left(2 Z_{o} \sqrt{\left.\left(g_{3} g_{4}\right)\right)}\right.}, \\
J_{45}=\frac{(\pi \Delta)}{\left(2 Z_{o} \sqrt{\left(g_{4} g_{5}\right)},\right.}, \text { and } J_{56}=\frac{\sqrt{(\pi \Delta)}}{\left(Z_{o} \sqrt{\left(2 g_{6} g_{7}\right)}\right)}
\end{gathered}
$$

(d) Even and odd mode impedance calculation: The even and odd mode characteristics line impedance is computed using:

$$
\begin{aligned}
& \left.Z_{\mathrm{o}_{\_} \text {odd }}\right|_{i, i+1}=Z_{\mathrm{o}}\left[1-Z_{\mathrm{o}} J_{i, i+1}+\left(Z_{\mathrm{o}} J_{i, i+1}\right)^{2}\right] \text {, and } \\
& \left.Z_{\mathrm{o} \_ \text {even }}\right|_{i, i+1}=Z_{\mathrm{o}}\left[1-Z_{\mathrm{o}} J_{i, i+1}+\left(Z_{\mathrm{o}} J_{i, i+1}\right)^{2}\right]
\end{aligned}
$$

where, $i, i+1$ refers to the overlapping of adjacent elements of coupled line filters. $Z_{0}$ is the characteristics line impedance at the beginning and end of the filter structure.

(e) Simulation using Linpar: Linpar [11] is a 2D Method of Moment (MoM) analysis package developed for numerical calculations involved in the design of microwave circuits and transmission lines. Using Linpar, for the specified dielectric constant and stripline dimensions, the separation $s$, and width $w$ of the copper strips are determined to meet the calculated even and odd mode impedances. Generally, the width conforms to the width of the other microstrip lines, therefore, the separation is the most common parameter that can be varied to achieve the even and odd mode impedances. The length of each coupled line segment has to be equal to $\lambda / 4$ at the centre frequency.

(f) Optimization of circuit parameters to meet the desired specifications: Once the design is completed, the circuit has to be simulated to observe the characteristic response. If the observed response deviates from the desired response, it is required to optimize the design by varying the input parameters within the acceptable range.

(g) Compensation for the length, width and spacing: The precise design can be achieved by introducing the length correction to account for fringing field effects. This factor can be calculated as:

$$
L_{e o}=0.412 h \frac{\left(\varepsilon_{\text {eff }}+0.3\right)\left(\frac{\mathrm{W}}{h}+0.264\right)}{\left(\varepsilon_{\text {eff }}-0.258\right)\left(\frac{\mathrm{W}}{h}+0.8\right)}
$$

where, $h$ is the height of the substrate or substrate thickness. $\varepsilon_{\text {eff }}$ is the effective dielectric constant of the substrate that varies with the length of the stripline, and $w$ is the width of the micro strip line. To account for the etching loss, compensation should be applied in terms of width and spacing, and hence, in the present work, the width is increased by factor $0.012 \mathrm{~mm}$, and so should decrease the spacing by the same factor. 


\section{RESULTS AND DISCUSSION}

\subsection{Design calculations and simulations}

(a) Calculation of $\varepsilon_{\text {eff }}$ : It involves simulation in Linpar, shown in Fig. 3, where, $t=0.017 \mathrm{~mm}$ is the input for a finite-thickness coupled microstrips above finite ground plane, and its $w$ is varied to attain $\varepsilon_{r}=10.5$ at $Z_{0}=50 \Omega$. The result of simulation revealed $\varepsilon_{e f f}=6.933$, and attenuation $=$ $1.976 \mathrm{~dB} / \mathrm{m}$. This section lists and explains the obtained research results. Also, it is desirable to compare the obtained results with other authors' results.

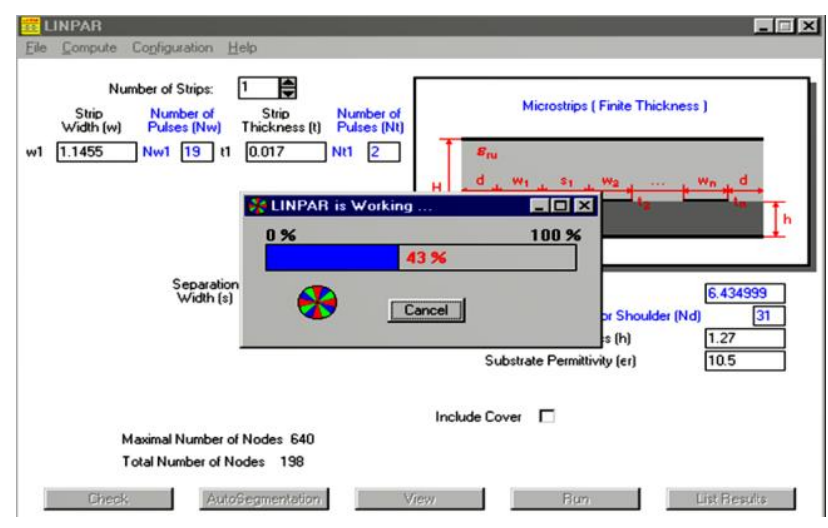

Fig. 3. Simulation for effective dielectric constant in Linpar

(b) Calculation of $\lambda_{o}$ and $\lambda / 4$ :

$$
\begin{aligned}
\lambda_{0} & =c / f_{\mathrm{o}}=66.7 \mathrm{~mm}, \text { and } \\
\lambda / 4 & =\lambda_{0} /\left(4 \sqrt{\left.\varepsilon_{\text {eff }}\right)}=6.33 \mathrm{~mm}\right.
\end{aligned}
$$

(c) Filter coefficient: The order of filter is 5 , so for the 5th order filter, the values of ' $g$ ' can be approximated from the standard table value for Chebyshev filter having $g_{0}=1, \omega_{\mathrm{c}}=1, n=5$, and 0.1 dB ripple.

(d) Coupling coefficients: Considering, fractional bandwidth $(\Delta)=0.1$, the coupling between the individual filter elements are obtained as $J_{01}=$ $0.0074, J_{12}=0.002504, J_{23}=0.001908, J_{34}=$ $0.001908, J_{45}=0.002504$, and $J_{56}=0.0074$.

(e) Even and Odd mode impedance: The calculated values of $Z_{o_{-} \text {odd }}$ and $Z_{o_{-} \text {even }}$ are tabulated in Table 2 .

Table 2. Even and Odd mode impedances

\begin{tabular}{|c|l|l|l|}
\hline$n$ & \multicolumn{1}{|c|}{$J_{n}$} & $Z_{\text {__even }}(\Omega)$ & $Z_{\text {o_odd }}(\Omega)$ \\
\hline 1 & 0.0074 & 75.345 & 38.345 \\
\hline 2 & 0.002504 & 57.044 & 44.524 \\
\hline 3 & 0.001908 & 55.225 & 45.685 \\
\hline 4 & 0.001908 & 55.225 & 45.685 \\
\hline 5 & 0.002504 & 57.044 & 44.524 \\
\hline 6 & 0.0074 & 75.345 & 38.345 \\
\hline
\end{tabular}

(f) Determination of stripline dimensions: As it is an edge-coupled filter, so the two lines are taken at a time and the values of $w$ and separation width $(s)$ are varied in Linpar to match with the value of $Z_{o_{-} \text {odd }}$ and $Z_{o_{-} \text {even }}$ (Fig. 4). The simulation output from Linpar provides the primary $[L],[C],[R]$ and [G] matrices for finite- thickness coupled microstrips above finite ground plane.

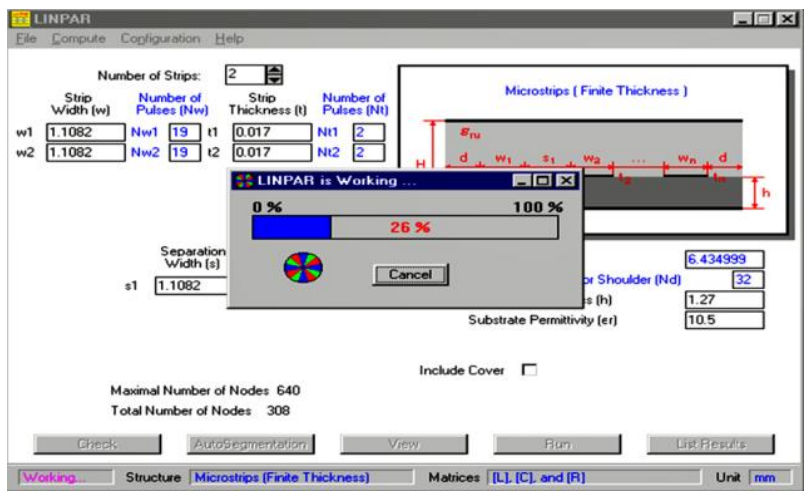

Fig. 4. Linpar simulations to attain the stripline dimensions

The circuit parameters of each coupling is obtained, as tabulated in Table 3. To account for the etching loss, compensation of $\pm 0.012 \mathrm{~mm}$ is applied in width and spacing.

Table 3: Detail of widths of final circuit obtained after simulation

\begin{tabular}{|c|c|c|c|}
\hline$n$ & $J_{n}$ & $\begin{array}{c}\text { Strip widths, } w_{1} \text { and } \\
w_{2}(\mathrm{~mm}) \text { after } \\
\text { compensation of } \\
+0.012 \mathrm{~mm}\end{array}$ & $\begin{array}{c}\text { Separation width, } s_{1} \\
(\mathrm{~mm}) \text { after } \\
\text { compensation of }- \\
0.012 \mathrm{~mm}\end{array}$ \\
\hline 1 & 0.0074 & 0.8161 & 0.34812 \\
\hline 2 & 0.002504 & 1.1007 & 1.42 \\
\hline 3 & 0.001908 & 1.1202 & 1.785 \\
\hline 4 & 0.001908 & 1.1202 & 1.785 \\
\hline 5 & 0.002504 & 1.1007 & 1.42 \\
\hline 6 & 0.0074 & 0.8161 & 0.34812 \\
\hline
\end{tabular}

\subsection{Fabrication and test results}

The layout of filter is prepared in CorelDraw 9.0 (Fig. 5a). The design of filter is realized on a high quality RT/ duroid 6010LM laminate having $\varepsilon_{r}=$ 10.5 and substrate thickness $1.27 \mathrm{~mm}$, as shown in Fig. $5 \mathrm{~b}$. The performance of the filter is checked on HP 8719D microwave network analyzer, and the achieved center frequency is $4.368 \mathrm{GHz}$ with passband between $3.86 \mathrm{GHz}$ to $4.95 \mathrm{GHz}$. The measured passband return loss (VSWR) achieved is $21 \mathrm{~dB}(1.196: 1)$. 


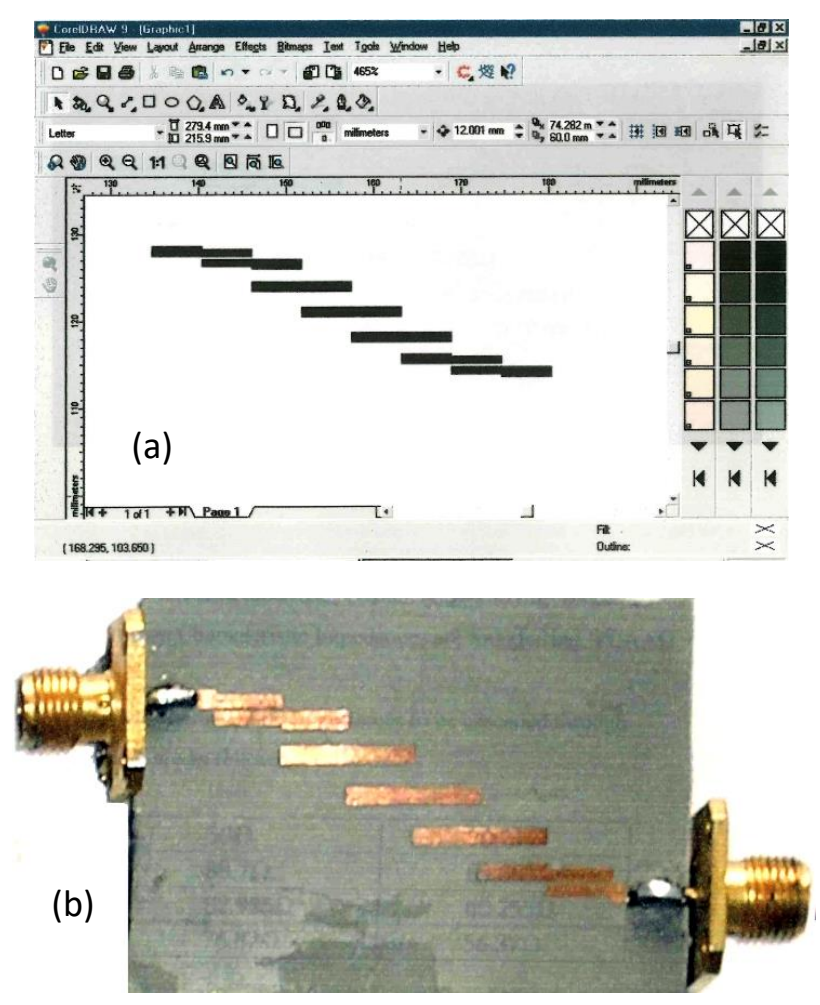

Fig. 5. (a) Filter circuit layout in CorelDraw, and (b) final fabricated circuit of edge-coupled bandpass filter

The center frequency and the filter's bandwidth have an offsets from the design value. These offsets are most likely a result of wide tolerance in the dielectric permittivity specified for the RT/ duroid substrate. Other possible causes of the offsets might have been the network analyzer calibration, impedance mismatch amongst the cables and adapters, or trace impedance errors occurred during the process of etching of the laminate.

\section{CONCLUSION}

The edge-coupled bandpass filter have been designed and fabricated using microstrip line and the performance is evaluated. Due to fabrication limitations some fabrication losses are introduced. By overcoming the fabrication problems these losses can be minimized, and the filter response can be improved.

\section{REFERENCES}

[1] R. Levy, R. V. Snyder, G. Matthaei, Design of microwave filters, Microwave Theory and Techniques. IEEE Transactions on, 50 (3), 2002: 783-793.

http://dx.doi.org/10.1109/22.989962

[2] I. C. Hunter, L. Billonet, B. Jarry, P. Guillon, Microwave filters-applications and technology, Microwave Theory and Techniques, IEEE Transactions on, 50 (3), 2002: 794-805.

http://dx.doi.org/10.1109/22.989963

[3] D. M. Pozar, Microwave Engineering. 2nd ed. Wiley. New York, 1998.

[4] Garg, R., Bahl, I.J., 1979. Characteristics of Coupled Microstriplines. IEEE Trans. Microw. Theory Tech., 27 (7), 1979: 700-705.

http://dx.doi.org/10.1109/TMTT.1979.1129704

[5] J. O. Scanlan, Theory of microwave coupledline networks. In Proceedings of the IEEE, 68 (2), 1980: 209-231.

http://dx.doi.org/10.1109/PROC.1980.11618

[6] L. G. Maloratsky, Reviewing the basics of microstrip lines. Microwave \& RF, 2000: 79-88.

[7] E. O. Hammerstad, O. Jensen, Accurate models for microstrip computer-aided design. IEEE MTT-S, Digest, 1980: 407-409.

http://dx.doi.org/10.1109/MWSYM.1980.1124303

[8] J. S. Hong, Microstrip Filter For RF/Microwave Application. John Willey and Sons, Inc, Second edition, 2011: 112-160.

[9] J. A. Ruiz-Cruz, C. Wang, K. A. Zaki, Design and characterization of Advances in microwave filter design techniques. Microwave Journal, 51 (11), 2008: 26-44.

[10] P. Gupta, A. Kumar, Design of Microwave Filter using Coupled Line Technique, Design and characterization of Advances in microwave filter design techniques, imanager's. Journal on Wireless Communication Networks, 5 (4), 2017: 27-31.

http://doi.org/10.26634/jwcn.5.4.13556

[11] A. R. Djordjevic, M. B. Bazdar, T. K. Sarkar, R. F. Harrington, LINPAR for Windows: Matrix Parameters for Multiconductor Transmission Lines. Artech House, 1995. 